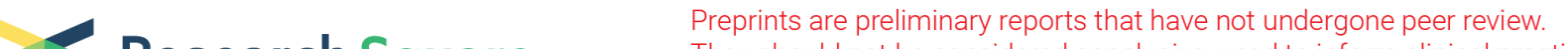 Research Square
or referenced by the media as validated information.
}

\section{TRF-Val-CAC-016 Modulates the Transduction of CACNA1d-Mediated MAPK Signaling Pathways to Suppress the Proliferation of Gastric Carcinoma}

\section{Weiguo Xu}

Jiangsu Cancer Hospital \& The Affiliated Cancer Hospital of Nanjing Medical University \& Jiangsu Institute of Cancer Research

\section{Junyu Zheng}

Jiangsu Cancer Hospital \& The Affiliated Cancer Hospital of Nanjing Medical University \& Jiangsu Institute of Cancer Research

\section{Xiao Wang}

Jiangsu Cancer Hospital \& The Affiliated Cancer Hospital of Nanjing Medical University \& Jiangsu Institute of Cancer Research

\section{Bin Zhou}

Jiangsu Cancer Hospital \& The Affiliated Cancer Hospital of Nanjing Medical University \& Jiangsu Institute of Cancer Research

\section{Huanqiu Chen}

Jiangsu Cancer Hospital \& The Affiliated Cancer Hospital of Nanjing Medical University \& Jiangsu Institute of Cancer Research

\section{Gang Li}

Jiangsu Cancer Hospital \& The Affiliated Cancer Hospital of Nanjing Medical University \& Jiangsu Institute of Cancer Research

\section{Feng Yan ( $\nabla$ yanfeng@jszlyy.com.cn)}

Jiangsu Cancer Hospital \& The Affiliated Cancer Hospital of Nanjing Medical University \& Jiangsu Institute of Cancer Research

\section{Research Article}

Keywords: tRNA derivative, tsRNA, immunoprecipitation, hierarchical clustering, post-transcriptional regulation

Posted Date: January 6th, 2022

DOl: https://doi.org/10.21203/rs.3.rs-1204437/v1 
License: (c) (i) This work is licensed under a Creative Commons Attribution 4.0 International License. Read Full License 


\section{Abstract}

Background: As a new kind of non-coding RNAs (ncRNAs), tRNA derivatives play an important role in gastric carcinoma (GC). Nevertheless, the underlying mechanism tRNA derivatives were involved in was rarely illustrated.

Methods: We screened out the tRNA derivative, tRF-Val-CAC-016, based on the tsRNA sequencing and demonstrated the effect tRF-Val-CAC-016 exerted on GC proliferation in vitro and in vivo. We applied Dualluciferase reporter assay, RIP assay, and bioinformatic analysis to discover the downstream target of tRFVal-CAC-016. Then CACNA1d was selected, and the oncogenic characteristics were verified. Subsequently, we detected the possible regulation of the canonical MAPK signaling pathway to further explore the downstream mechanism of tRF-Val-CAC-016.

Results: As a result, we found that tRF-Val-CAC-016 was low-expressed in GC, and upregulation of tRFVal-CAC-016 could significantly suppress the proliferation of GC cell lines. Meanwhile, tRF-Val-CAC-016 regulated the canonical MAPK signaling pathway by targeting CACNA1d.

Conclusions: tRF-Val-CAC-016 modulates the transduction of CACNA1d-mediated MAPK signaling pathways to suppress the proliferation of gastric carcinoma. This study discussed the function and mechanism of tRF-Val-CAC-016 in GC for the first time. The pioneering work has contributed to our present understanding of tRNA derivative, which might provide an alternative mean for the targeted therapy of GC.

\section{Background}

More than $70 \%$ of new cases of gastric cancer (GC) occurred in developing countries, and about $50 \%$ of GC cases occurred in eastern Asia, mainly in China. The number of incidences and deaths of gastric cancer in China accounted for $42.6 \%$ and $45.0 \%$ of the global statistical data, respectively, ranking 5 th in morbidity and 6th in mortality among 183 countries globally [1]. Therefore, it is essential to explore the treatment approaches for GC.

tsRNAs, as a new kind of discovered ncRNAs, have attracted increasing attention in cancer research. And tsRNAs were classified into tRFs and tiRNAs based on the cleavage sites of tRNAs by the ribonucleases such as Dicer and RNase Z [2, 3]. Meanwhile, with the illustration for the fundamental structures of tRFs and tiRNAs, it aroused great interest of many researchers in the aspect of biological characteristics and potential therapeutic strategies of tsRNAs in several kinds of carcinomas [4-8]. tsRNAs have been demonstrated as mRNA regulators by binding to the 3'UTR of specific mRNAs, similar to miRNAs [9].

CACNA1d has been verified as an oncogene in several pieces of literature [10-12]. And it was involved in the MAPK signaling pathways to regulate the proliferation of certain carcinomas [13]. As one of the calcium voltage-gated channel subunits, $C A C N A 1 d$ also played a role in the modulation of calcium ion concentration inside and outside the cells [14]. Furthermore, the MAPK signaling pathways was widely 
accepted as the canonical pathway to regulate the progression of GC in many types of researches [1519]. The bioinformatic analysis indicated that CACNA1d was located in the cell membrane and participated in the MAPK signaling pathways, consistent with a few previous researches $[13,20]$.

Concerning the present study, we screened out the tRF-Val-CAC-016 as the research target, and it was significantly down-regulated in GC tissues. Furthermore, we found MAPK signaling pathways and CACNA1d were regulated by tRF-Val-CAC-016. Therefore, we hypothesize that tRF-Val-CAC-016 modulates the transduction of $C A C N A 1 d$-mediated MAPK signaling pathways to suppress the proliferation of gastric carcinoma.

\section{Methods}

\section{Clinical samples}

All GC samples were collected in Jiangsu Cancer Hospital with informed consents from these patients. Meanwhile, this study was appraised and authorized by the ethics committee of Jiangsu Cancer Hospital (NYDLS-2019-919). All samples were snap-frozen in the refrigerator at $-80^{\circ} \mathrm{C}$, and the Declaration of Helsinki was obeyed.

\section{tRFs and tiRNAs sequencing profiles}

We screened out the tRFs and tiRNAs based on the fold-change (FC, cutoff 1.5) of expression data and pvalue (cutoff 0.05 ) of differentially expressed tsRNAs. Hierarchical clustering, Volcano plots, and Correlation Analysis were then used to present the essential data. GO and KEGG analysis was then conducted to sieve the significant target genes and signaling pathways. Subsequently, we applied the Gene Set Enrichment Analysis (GSEA) further to illustrate the potential characteristics of selected target genes. In addition, to comprehensively verify the expression levels and prognostic data of selected genes, we downloaded the GC-related data in TCGA (TCGA-STAD) and GEO databases (GSE65801). Finally, we imported all bioinformatic data into R software to select and verify the target genes.

\section{Cell culture and transfection}

We obtained GC cell lines NCI-N87 and HGC-27 from the Cell Bank of the Chinese Academy of Sciences (Shanghai, China), and were authenticated to be free from mycoplasma infections. NCI-N87 was cultured in the RPMI-1640 medium with 15\% FBS, HGC-27, SNU-216, BGC-823, and GES-1 in the RPMI-1640 medium $10 \%$ FBS, AGS was cultivated in the F-12K medium with $10 \%$ FBS. All cell lines were incubated in a humidified incubator at $37^{\circ} \mathrm{C}$ with $5 \% \mathrm{CO}$. We used lipofectamine2000 (Invitrogen, USA) to transfect $\mathrm{NCl-N87}$ and HGC-27. Transfection concentration was $50 \mathrm{nM}$ for tRF-Val-CAC-016 mimics, mimic control, siRNA-CACNA1d and siRNA-control, 100nM for tRF-Val-CAC-016 inhibitor and inhibitor control, $1.0 \mu \mathrm{g} / \mathrm{mL}$ for pcDNA-CACNA1d and pcDNA vector. We applied p38 MAPK-IN (MCE, USA) as the inhibitor of the MAPK signaling pathway. 


\section{RNA isolation, reverse transcription, and RT-PCR}

Total RNA was extracted with Trizol reagent (Life Technologies, USA), then the reverse transcription (RT) was performed with the riboSCRIPT Reverse Transcription Kit (RiboBio, Guangzhou, China), and the detailed protocols of the manufacturer were followed $\left(60 \mathrm{~min}\right.$ at $42^{\circ} \mathrm{C}$ and $10 \mathrm{~min}$ at $\left.70^{\circ} \mathrm{C}\right)$. We applied the LightCycler 1.5 (Roche, Switzerland) to perform the PCR reaction.

\section{Fluorescent In Situ Hybridization (FISH)}

Cell fixation was performed with $4 \%$ paraformaldehyde, and the fixed cells were then permeabilized for 5 min at $4^{\circ} \mathrm{C}$ with Triton X-100 (Beyotime, Shanghai, China). Subsequently, pre-hybridization, hybridization, and blocking were undertaken with the Ribo ${ }^{\mathrm{TM}}$ Fluorescent In Situ Hybridization Kit (RiboBio, Guangzhou, China). Finally, the cells nuclei were stained blue with 4', 6-diamidino-2-phenylindole (DAPI), and cytoplasm was stained red with the Cy3-labelled tRF-Val-CAC-016 (GenePharma, Shanghai, China).

\section{Cell proliferation assays}

In the CCK-8 assay, $100 \mu \mathrm{L}$ cell suspension containing 1000 cells was seeded in each well of 96-well plates, then $10 \mu \mathrm{L}$ CCK-8 reagent (Dojindo, Laboratories, Japan) was added into each well and incubated for two hours. We then measured the OD value in $450 \mathrm{~nm}$ by the microplate reader at $0,24,48,72$, and 96 hours. In the cell colony formation assay, $2 \mathrm{ml}$ cell suspension containing 4000 processed cells were seeded into each well of 6-well plates. After the incubation for 10-14 days, the cells were fixed with $4 \%$ paraformaldehyde for 20 minutes at room temperature (RT) and were then stained with $1 \%$ crystal violet. Images were captured with a microscope, and the number of colonies was counted with Image $\mathrm{J}$ software.

\section{Cell cycle determination}

We harvested the transfected cells and resuspended them in $70 \%$ ethanol. Afterward, cells were centrifuged, and the cell pellets were resuspended in PI-solution to conduct the flow cytometry analysis.

\section{EdU assay}

Ethynyl-2'-deoxyuridine (EdU) assay was undertaken with the Cell-Light EdU Apollo In Vitro Kit (Ribobio, Guangzhou, China). Each well of 96-well plates was seeded with $4 \times 10^{3}-1.0 \times 10^{5}$ cells, and these GC cells were then incubated in the EdU solution $(50 \mu \mathrm{M})$ for two hours. Subsequently, the cell fixation was performed with $4 \%$ paraformaldehyde, and cells were then washed with $50 \mu \mathrm{L}$ glycine $(2 \mathrm{mg} / \mathrm{mL})$. Analogously, we added $100 \mu \mathrm{L}$ penetrant $(0.5 \%$ TritonX-100) into each well to incubate these cells for 10 minutes. Next, we pipetted $1 \times$ Apollo reagent $(100 \mu \mathrm{L})$ into each well to initiate the staining reaction. Nuclei were stained with DAPI or $1 \times$ Hoechst33342, and images were captured with a fluorescence microscope.

\section{Dual-luciferase reporter assay}


GC cells were co-transfected with plasmids WT-CACNA1d-3'UTR or MUT-CACNA1d-3'UTR, and tRF-ValCAC-016 mimics or mimic control with lipofectamine2000 (Invitrogen, USA). We detected the luciferase activities of firefly and renilla with the Dual-Luciferase Reporter Assay System (Promega, Madison, USA). To begin with, we obtained the cell lysates using the Passive Lysis Buffer. After preparing Luciferase Assay Reagent II (LAR II) and Stop \& Glo Reagent, we carefully transferred $20 \mu$ l of cell lysate into the luminometer tube containing LAR II to record the firefly luciferase activity measurement. Then we added $100 \mu l$ of Stop \& Glo Reagent to record the renilla luciferase activity measurement.

\section{RNA-binding protein immunoprecipitation (RIP)}

We purchased the Magna RIP ${ }^{\text {TM }}$ RNA-Binding Protein Immunoprecipitation Kit (Merck, Darmstadt, Germany). First, we lysed GC cells in RIP lysis buffer and dispensed $200 \mu \mathrm{L}$ each of the lysates into nuclease-free microcentrifuge tubes and stored them at $-80^{\circ} \mathrm{C}$. Second, we incubated the washed beads with Argonaute-2 antibody to produce the beads-antibody complex and used the Normal Rabbit IgG antibody as the negative control. Third, $100 \mu \mathrm{L}$ supernatant of the RIP lysate was removed and added to each beads-antibody complex in RIP Immunoprecipitation Buffer $(10 \mu \mathrm{L}$ of the supernatant was stored at $-80^{\circ} \mathrm{C}$ as Input). All the tubes were incubated and rotated overnight at $4{ }^{\circ} \mathrm{C}$. Finally, RNA purification and RT-PCR were manipulated to test the binding tRF-Val-CAC-016.

\section{Immunohistochemistry (IHC) and immunofluorescence staining}

Tissue sections were deparaffinized and dehydrated, and then the tissues were blocked with serum and placed in the $37^{\circ} \mathrm{C}$ incubator for half an hour. Next, We added the primary antibodies to the slides and incubated them overnight in the $4^{\circ} \mathrm{C}$ incubator. Analogously, we then added the secondary antibodies to the sections and set them at $37^{\circ} \mathrm{C}$ for half an hour. Afterward, we stained these tissue sections with $D A B$ substrate and hematoxylin successively and dehydrated them with ethanol. Images were captured with a microscope. In terms of immunofluorescence staining, cells were rinsed and rewarmed with PBS for 20 minutes. We then applied $0.5 \%$ TritonX-100 to permeabilize the sections at RT for 20 minutes.

Subsequently, serum blocking and antibody incubation were performed successively. Nuclei were stained with DAPI. We collected images using a fluorescence microscope.

\section{Subcutaneous xenograft experiments}

Eighteen five-week-old Balb/c female nude mice were purchased from the Shanghai Experimental Animal Center of the Chinese Academic of Sciences (Shanghai, China) and were divided into tRF-Val-CAC-016 agomir group, tRF-Val-CAC-016 negative control (NC) group, and normal saline (NS) group randomly and evenly. These reagents were purchased from Ribobio (Ribobio, Guangzhou, China). Approximately $1.2 \mathrm{x}$ $10^{6}$ processed NCI-N87 cells were then subcutaneously injected into the right flanks of these mice. Tumor sizes and weights were measured every three days. After 39 days of rearing, all mice were sacrificed, and all tumors were resected. Tumor volumes were calculated with this formula: $1 / 2 \times\left(\right.$ length $\times$ width $\left.^{2}\right)$. All animal experiments complied with the National Research Council's Guide for the Care and Use of 
Laboratory Animals. The care of animals was in accordance with the institution guidelines of Jiangsu Cancer Hospital.

\section{Immunoblotting}

Gel-electrophoresis (10\% SDS-PAGE) for extracted proteins was conducted, and the proteins were then transferred to a polyvinylidene difluoride membrane. Next, we blocked the membranes with $1 \times$ Trisbuffered saline (TBS) containing $0.1 \%$ Tween 20 and $5 \%$ BSA or $5 \%$ skim milk for one hour at RT. Then, we incubated the membranes with specific primary antibodies overnight at $4^{\circ} \mathrm{C}$ and the secondary antibodies for two hours at RT. After three washes, the membranes were visualized with the chemiluminescence (ECL) kit (Millipore, Bedford, MA, USA). Basic information on the antibodies used in this research is presented in Supplementary Table 1.

\section{Statistical Analysis}

We utilized GraphPad Prism Version 4 program (GraphPad Software Inc., San Diego, CA) and SPSS 19.0 (IBM, Chicago, USA) to deal with the data. All numeric values were presented as the mean \pm SD. The significant data was determined using ANOVA followed by Dunnett's test or Student's unpaired t-test. $\mathrm{P}<$ 0.05 was taken as statistically significant.

\section{Results}

\section{tRFs and tiRNAs sequencing profiles of GC tissues}

We performed tsRNAs sequencing to distinguish the differentially expressed tsRNAs (DETs) and not differentially expressed tsRNAs (NDETs), and tRF-Val-CAC-016 was finally selected based on the profiles. And then, we applied the hierarchical clustering to classify the DETs, a total of 69 up-regulated and 42 down-regulated DETs were presented with hierarchical clustering heatmap (Fig. 1a). We used the volcano plot to exhibit DETs of great significance, which indicated that five down-regulated and six up-regulated tsRNAs were presented in the plot based on the foldchange $(F C)$ of the profiles $\left(\log _{2} F C>=3\right.$ or $\log _{2} F C<=-3$, p<0.05) (Fig. 1b). Obviously, we selected tRF-Val-CAC-016 for further research considering the feasibility and statistical significance. Meanwhile, we accomplished the heatmap of the correlation coefficient to elaborate on the similarity of the GC samples using the R gplots package (Fig. 1C). The distribution and frequency of tsRNA subtypes were presented in Supplementary Fig. 1a-b and Supplementary Fig. 1c-d, respectively. The classification of tsRNA isodecoders is shown in Supplementary Fig. 1ef.

\section{tRF-Val-CAC-016 was significantly low-expressed in GC tissues.}

We applied Gel-electrophoresis to verify the PCR product of tRF-Val-CAC-016, demonstrate the feasibility of PCR primers, and verify the authenticity of tsRNA sequencing (Fig. 2a). As shown in Fig. 2b, the length of tRF-Val-CAC-016 ranged between $50 \mathrm{bp}$ and $100 \mathrm{bp}$, and we undertook the Sanger sequencing to verify the results. Then Fluorescent In Situ Hybridization (FISH) assay indicated that tRF-Val-CAC-016 is located 
in both nuclei and cytoplasm, but mainly in the cytoplasm (Fig. 2c). Afterward, the expression level of tRFVal-CAC-016 was tested in GC cell lines, NCl-N87 and HGC-27 were finally selected (Fig. 2d). Finally, to confirm the efficiency of tRF-Val-CAC-016 mimics, we conducted the transfection, and the result was in line with our expectations (Fig. 2e). Analogously, the low expression level was confirmed in 40 pairs of GC tissues (Fig. 2f). And the expression of tRF-Val-CAC-016 was significantly associated with tumor size and histology in the aspect of clinicopathological features (Table 1). Furthermore, the prognostic outcome of tRF-Val-CAC-016 was calculated after the follow-up for the patients after gastrectomies, but the result was not significant (Fig. $\mathbf{2 g}$ ). The sequences of tRF-Val-CAC-016 and related primers are listed in Supplementary Table 2.

\section{tRF-Val-CAC-016 suppressed the proliferation of GC.}

As shown in Fig. 3a-b, tRF-Val-CAC-016 significantly suppressed the proliferation of GC cells in CCK-8 assays, achieving a similar performance of oxaliplatin. Besides, tRF-Val-CAC-016 inhibitor promoted the proliferation of GC compared with the inhibitor control. The ethynyl-2'-deoxyuridine (EdU) assays demonstrated that tRF-Val-CAC-016 could suppress the cell replication activity of GC, but tRF-Val-CAC-016 inhibitor enhanced the replication activity (Fig. 3c-d). Meanwhile, we found that tRF-Val-CAC-016 could regulate the checkpoints of the cell cycle in GC. As presented in Fig. 3e-g, oxaliplatin mainly handled the G1 phase in GC. However, tRF-Val-CAC-016 adjusted the S phase significantly both in NCI-N87 and HGC27. In colony formation assays, tRF-Val-CAC-016 could inhibit the viability of GC cells, slightly weaker than oxaliplatin. tRF-Val-CAC-016 inhibitor enhanced the ability of colony formation in GC cells (Fig. 3h-i). Consistently, these phenomena were rigorously explained in the immunoblotting assays, as indicated in Fig. 3j-k, tRF-Val-CAC-016 mimics obviously declined the protein expression of CyclinD1, CyclinB, c-myc. tRF-Val-CAC-016 inhibitor increased the protein expression of CyclinD1, CyclinB, c-myc. On the other hand, oxaliplatin decreased the expression of CyclinD1, c-myc compared with the control.

\section{Bioinformatics analysis}

Target genes of the down-regulated or the up-regulated tsRNAs were then enriched in the GO and KEGG analysis. In the down-regulated group, GO analysis was presented in Fig. 4a-c, andwe found that the MAPK signaling pathways were enriched significantly (Fig. 4d). In the up-regulated group, GO analysis was shown in Fig. 4e-g, andwe found that the Wnt signaling pathway was quite prominent (Fig. 4h). We then compared the bioinformatics data in the present study with GEO (Supplementary Fig. 2) and TCGA databases (Supplementary Fig. 3), which suggested that proliferation-related pathways were frequently enriched (Supplementary Fig. $\mathbf{2 f}$ and Supplementary Fig. $\mathbf{3 f}$ ), and the Calcium signaling pathway was uncovered (Supplementary Fig. 3i), consistent with the function of CACNA1d.

CACNA1d was verified up-regulated in GC tissues and was selected as the potential target of tRF-Val-CAC016.

We introduced the TCGA and GEO databases to predict the possible target to conduct further research. As shown in Fig. 5a, the Venn diagram took the overlap of MAPK components and the predicted target genes 
of tRF-Val-CAC-016. Then we analyzed the GEO database (GSE65801) and TCGA-STAD database and found that CACNA1d, PLA2G4A and TNF in GSE65801 (Fig. 5b), CACNA1d and PLA2G4A inTCGA-STAD were significantly up-regulated (Fig. 5c). Furthermore, we then applied the Kaplan-Meier plotter website and discovered that CACNA1d, TNF, TGFBR1, PDGFC, GADD45B were significantly and oppositely related to the prognosis of GC (Fig. $5 \mathrm{~d}-\mathrm{l})$. Analysis above reminded us the vital role of CACNA1d as the possible downstream target of tRF-Val-CAC-016. Subsequently, we obtained the tissue microarray (TMA) with 90 pairs of GC specimens, including detailed follow-up data (Fig. 5m). IHC results were presented in Fig. $\mathbf{5 n}$. Through the analysis of the follow-up data, we found that the expression of CACNA1d was not significantly related to the prognosis of GC $(p=0.1805)$ (Fig. 5o). The representative IHC images of GC and NATs are shown in Fig. 5p.However, the protein levels of CACNA1d in GC tissues were up-regulated compared with corresponding NATs (Fig. 5q). Hence, we selected CACNA1d as the target gene based on the intersection of the comprehensive analysis. To confirm the expression and function of CACNA1d, we purchased siRNAs for $C A C N A 1 d$ and selected si-CACNA1d-1 as the better inhibitory effect compared with si-CACNA1d-2 and si-CACNA1d-3 (Fig. 5r). On the contrary, pcDNA-CACNA1d couldsignificantly enhance the expression of CACNA1d (Fig. 5s). Moreover, the tRF-Val-CAC-016 inhibitor was able to reverse the suppressive function of si-CACNA1d on GC cells to some extent (Fig. 5t). Analogously, the effect of pcDNA-CACNA1d on GC was partly relieved by tRF-Val-CAC-016 mimics (Fig. $5 \mathrm{u}$ ). The sequences of CACNA1d primers and siRNAs are listed in Supplementary Table 2.

\section{tRF-Val-CAC-016 was immunoprecipitated by Argonaute-2 and could modulate the proliferation of GC by targeting CACNA1d.}

The sequencing profile has elucidated the possible binding relation between tRF-Val-CAC-016 and CACNA1d mRNA (Fig. 6a), and the tRF-Val-CAC-016 mimics could significantly reduce the expression of CACNA1d in RT-PCR (Fig. 6b). Theprotein level of CACNA1d was decreased by tRF-Val-CAC-016 mimics and promoted by tRF-Val-CAC-016 inhibitor (Fig. 6c). Subsequently, we found that WT-CACNA1d-3'UTR plus tRF-Val-CAC-016 mimics group could significantly reduce the luciferase ratio compared with other groups in the Dual-luciferase reporter assay (Fig. 6d). Then we introduced RIP (RNA-binding protein immunoprecipitation) assay and found that tRF-Val-CAC-016 was significantly immunoprecipitated by Argonaute-2 compared with the IgG group (Fig. 6e). Gel-electrophoresis further verified the PCR product of RIP assays (Fig. 6f). And the immunoblotting confirmed the integrity of the process to wash the magnetic beads (Fig. 6g).

\section{CACNA1d strengthened the proliferation of GC and was modulated by tRF-Val-CAC-016.}

Rescue assays were undertaken to elucidate the connection between tRF-Val-CAC-016 and CACNA1d further. In the CCK-8 assays, si-CACNA1d could significantly decline the proliferation of GC cells, which was partially recovered by tRF-Val-CAC-016 inhibitor (Fig. 7a-b). In the EdU assays,the tRFVal-CAC-016 inhibitor could rescue the inhibitory effect of si-CACNA1d in terms of cell replication activity (Fig. 7c-d). Interestingly, si-CACNA1d resulted in the $\mathrm{G} 1$ phase arrest in HGC-27 but $\mathrm{S}$ phase arrest in $\mathrm{NCl}-$ N87, and could both be rescued by tRF-Val-CAC-016 inhibitor (Fig. 7e-g). Similarly, the rescue effect of the 
tRF-Val-CAC-016 inhibitor on si-CACNA1d was also confirmed in the colony formation assays (Fig. 7h-i). The suppressive function of si-CACNA1d on the protein expression of CACNA1d, CyclinD1, CyclinB, c-myc was partly resumed by tRF-Val-CAC-016 inhibitor (Fig. 7j-k). Analogously, the reverse verification for the function of CACNA1d was then performed. pcDNA-CACNA1d and pcDNA-CACNA1d plus tRF-Val-CAC-016 mimics were transfected, respectively. As shown in Fig. 8a-i, tRF-Val-CAC-016 mimics could significantly reverse the reinforced effect of pcDNA-CACNA1d on the proliferation of GC cells. Moreover, pcDNACACNA1d significantly lifted the protein levels of CACNA1d, CyclinD1, CyclinB, c-myc, but tRF-Val-CAC016 mimics could decline this function (Fig. 8j-k).

\section{Inhibitor of MAPK signaling pathway (p38 MAPK-IN) significantly reversed the enhancement of tRF-Val- CAC-016 inhibitor on GC proliferation.}

To demonstrate the role of the MAPK signaling pathway, we took p38 MAPK-IN to rescue the enhancement of tRF-Val-CAC-016 inhibitor on GC proliferation. It indicated that p38 MAPK-IN was able to block the pathway conduction partly as expected, which was manifested in the aspect of the deterioration to the influence of tRF-Val-CAC-016 inhibitor on GC. As shown in Fig. 9a-b, tRF-Val-CAC-016 inhibitor enhanced the GC proliferation in the CCK-8 assay, which p38 MAPK-IN could partly reverse. Meanwhile, tRF-Val-CAC-016 inhibitor promoted the cell replication activity, and p38 MAPK-IN could also decline this activity (Fig. 9c-d). The reversal phenomena also happened in the flow cytometry assays for cell cycle (Fig. 9e-g), and immunoblotting assays for CACNA1d, CyclinD1, CyclinB, c-myc (Fig. 9h-i).

\section{tRF-Val-CAC-016 suppressed tumor proliferation in NCI-N87 xenografts.}

Subcutaneous xenograft experiments were undertaken to discuss the function of tRF-Val-CAC-016 in vivo. The results indicated that tRF-Val-CAC-016 declined the capacity of tumor growth in mice in the aspect of the bodyweight of the xenografts and the tumor volumes (Fig. 10a-d). The PCR results of these resected tumors showed that tRF-Val-CAC-016 agomir significantly suppressed the proliferation compared with the agomir control group and normal saline (NS) group (Fig. 10e), which also happened in the immunoblotting assays for these mice tumors (Fig. 10f). Besides, the immunofluorescence staining assays for ki-67 and CACNA1d suggested that tRF-Val-CAC-016 reduced the proliferative capacity of GC and further confirmed the expression and location of CACNA1d protein (Fig. 10g-h). Meanwhile, thelmmunohistochemistry (IHC) assays also demonstrated the low expression of CACNA1d in the tRFVal-CAC-016 agomir group (Fig. 10i).

\section{tRNA derivative tRF-Val-CAC-016 modulates the canonical MAPK signaling pathways by targeting CACNA1d.}

As presented in Fig. 11a, the mechanism diagram clearly explained the pattern that tRF-Val-CAC-016 exerted its influence on the MAPK signaling pathway. Overview of MAPK signaling pathway was shown in Supplementary Fig. 4. In the immunoblotting assays of NCI-N87, tRF-Val-CAC-016 mimics suppressed the expression of CACNA1d (Cav1.3), ERK, p-ERK, and p-p38. tRF-Val-CAC-016 inhibitor enhanced the expression of CACNA1d (Cav1.3), ERK, p-JNK, p38, cyclinB, cyclinD1, c-myc (Fig. 11b). si-CACNA1d could 
inhibit the expression levels of CACNA1d (Cav1.3), JNK, p-p38, cyclinD1, and c-myc, which was able to be reversed by tRF-Val-CAC-016 inhibitor (Fig. 11c). pcDNA-CACNA1d increased the expression of CACNA1d (Cav1.3), ERK, p-ERK, p-JNK, p38, p-p38, cyclinB, cyclinD1, and c-myc, which was rescued by tRF-Val-CAC016 mimics (Fig. 11d). In HGC-27, tRF-Val-CAC-016 mimics declined, but tRF-Val-CAC-016 inhibitor promoted the protein levels of CACNA1d (Cav1.3), ERK, p-ERK, JNK, p-JNK, p38, p-p38, cyclinB, cyclinD1, c-myc (Fig. 11e). And CACNA1d (Cav1.3), ERK, JNK, p-JNK, p38, p-p38, cyclinB, and cyclinD1 were downregulated by si-CACNA1d, and this effect was reversed by tRF-Val-CAC-016 inhibitor (Fig. 11f).

CACNA1d (Cav1.3), ERK, p-ERK, JNK, p-JNK, p38, p-p38, cyclinB, cyclinD1, and c-myc were enhanced by pcDNA-CACNA1d, which could be deteriorated by tRF-Val-CAC-016 mimics (Fig. 11g).

\section{Discussion}

tsRNAs are a class of ubiquitously expressed and conserved ncRNAs in nature. Emerging researches suggest that tsRNAs have been a rising star in this field of cancer research [7, 21-28].

The results of a considerable amount of researches were consistent with our present study regarding the role of tsRNAs as a regulator in the aspect of tumor progression [7, 29-37]. Farina et al. found that RUNX1 could maintain the mammary epithelium by repressing ts-112 [38]. Kim et al. discovered that LeuCAG3'tsRNA might maintain ribosomal protein S28 (RPS28) levels after translation initiation to explain why some mRNA levels do not necessarily correlate with protein levels [39]. Wang et al. identified the novel tsRNA-06018 as a critical regulator in hMSC adipogenesis [40]. Generally speaking, a considerable amount of researches mainly focused on the post-transcriptional regulation to influence the translation of specific proteins. However, many researchers also found the critical function of tsRNAs as the biomarkers in diagnosing diseases [5, 41, 42]. Meanwhile, many tsRNAs were found related to several signaling pathways to facilitate the modulation of certain tumors' progression. For instance, Wang et al. reported that tiRNA ${ }^{\text {Tyr-GTA }}$ could modulate the peroxisome proliferator-activated receptor signaling pathway [43]. Furthermore, tRF/miR-1280 was discovered to maintain the function of cancer stem celllike cells of CRC by inhibiting the Notch signaling pathway [24]. Balatti et al. applied CRISPR technology to generate ts-101 and ts-46 KO stable cell lines from HEK293 cells, then Affymetrix gene-expression profiling was performed to screen the differentially expressed genes. Finally, they discovered that tsRNAs could regulate the chromatin configuration and the epigenetic control of gene expression. In addition, several signaling pathways related to cell transformation and cancer development, such as PDGF signaling, mTOR signaling, and PTEN signaling, were also found to be modulated [44]. Our study demonstrated the critical role of tRF-Val-CAC-016 in the activation of the MAPK signaling pathway, which was also noticeable in the field of tsRNAs research.

In terms of the present research, we selected tRF-Val-CAC-016 as the research target. Interestingly, tRF-ValCAC-016 was significantly low-expressed in GC cell lines (NCI-N87 and HGC-27) and tissues compared with GES-1 and NATs, respectively. By the overexpression of tRF-Val-CAC-016, we observed that tRF-ValCAC-016 could suppress the proliferation of GC cells. Through the RIP assay and Dual-luciferase reporter assay, we rigorously confirmed the bioinformatic results concerning the binding relation between tRF-Val- 
CAC-016 and CACNA1d. As CACNA1d is a member of the MAPK signaling pathway, we assumed that tRFVal-CAC-016 might modulate the GC proliferation via tRF-Val-CAC-016/ CACNA1d/MAPK signaling pathways axis. Through the rescue assays, we confirmed the regulative relationships among tRF-ValCAC-016, CACNA1d, and MAPK signaling pathway. And we conducted the subcutaneous xenograft experiments to verify the function of tRF-Val-CAC-016 in vivo further. Subsequently, the results of immunoblotting assays related to the MAPK signaling pathway were consistent with our hypothesis that tRF-Val-CAC-016 modulates the canonical MAPK signaling pathways by targeting CACNA1d to influence the proliferation of gastric carcinoma. Hence, these findings uncovered a remarkable role of tRF-Val-CAC016 as a tumor inhibitor in GC.

This study preliminarily explored the mechanism that tRF-Val-CAC-016 participated in to regulate the proliferation of GC. However, there still exist a few deficiencies in this research. For instance, the detailed mechanism of tRNAs splicing to produce tsRNAs was not investigated by us thoroughly. And the regulatory network of the significant tsRNAs is not analyzed comprehensively. The crosstalking among the tsRNAs needs to be discovered in further research.

\section{Conclusions}

In summary, this research illustrated the function of tRF-Val-CAC-016 in GC for the first time. And the discussion for the mechanism tRF-Val-CAC-016 was involved in was relatively comprehensive to some extent. This study showed that tRF-Val-CAC-016 was down-regulated significantly in GC, and the expression was related to the histology and tumor size in terms of the clinicopathological analysis. Besides, tRF-Val-CAC-016 modulates the transduction of CACNA1d-mediated MAPK signaling pathways to suppress the proliferation of gastric carcinoma. Taken together, tRF-Val-CAC-016 could be a potential diagnostic biomarker and a new therapeutic target for GC.

\section{Abbreviations}

GC: Gastric Cancer; TNM: Tumor-Node-Metastasis; RT-PCR: Real time-PCR; GSEA: Gene Set Enrichment Analysis; GO: Gene Ontology; KEGG: Kyoto Encyclopedia of Genes and Genomes; NATs: Non-tumor Adjacent Tissues; DETs: Differentially Expressed tsRNAs; NDETs: Not Differentially Expressed tsRNAs; RIP: RNA-binding protein immunoprecipitation; EdU: Ethynyl-2'-deoxyuridine; FISH: Fluorescent In Situ Hybridization; RT: Room Temperature; NS: Normal Saline; FC: Fold-Change.

\section{Declarations}

\section{Author contributions}

WX, FY, and HC: conception and design. WX, JZ, XW, and BZ: acquisition of data. WX, and JZ: analysis and interpretation of data. WX: writing and review of the manuscript. FY and GL: study supervision. All authors have contributed to the article and approved the submitted version. 
Acknowledgements

We thank all members of our group for discussion and assistance with this study. This research was supported by the National Natural Science Foundation of China (NO. 81871718); Jiangsu Commission of Health Surface Project (NO. H2017034); the National Key Research and Development Program (NO. 2017YFC0908300), Jiangsu Provincial Key Research and Development Program (NO. BE2018750), a grant from the General Program of Jiangsu cancer hospital (NO. ZM202003).

\section{Ethics approval}

All procedures performed in studies involving human participants were in accordance with ethical standards of the institutional and/or national research committee and with the 1964 Helsinki Declaration and its later amendments or comparable ethical standards.

\section{Consent for publication}

Not applicable.

\section{Availability of data and materials}

The datasets used and/or analysed during the current study are available from the corresponding author on reasonable request.

\section{Conflicts of interest}

These authors declare no conflicts of interest.

\section{References}

1. Chen W, Zheng R, Baade PD, Zhang S, Zeng H, Bray F, Jemal A, Yu XQ, He J: Cancer statistics in China, 2015. CA Cancer J Clin 2016, 66:115-132.

2. Cole C, Sobala A, Lu C, Thatcher SR, Bowman A, Brown JW, Green PJ, Barton GJ, Hutvagner G: Filtering of deep sequencing data reveals the existence of abundant Dicer-dependent small RNAs derived from tRNAs. Rna 2009, 15:2147-2160.

3. Lee YS, Shibata Y, Malhotra A, Dutta A: A novel class of small RNAs: tRNA-derived RNA fragments (tRFs). Genes Dev 2009, 23:2639-2649.

4. Fagan SG, Helm M, Prehn JHM: tRNA-derived fragments: A new class of non-coding RNA with key roles in nervous system function and dysfunction. Prog Neurobiol 2021, 205:102118.

5. Gu W, Shi J, Liu H, Zhang X, Zhou JJ, Li M, Zhou D, Li R, Lv J, Wen G, et al: Peripheral blood noncanonical small non-coding RNAs as novel biomarkers in lung cancer. Mol Cancer 2020, 19:159.

6. Wang T, Mei J, Li X, Xu X, Ma B, Li W: A novel tsRNA-16902 regulating the adipogenic differentiation of human bone marrow mesenchymal stem cells. Stem Cell Res Ther 2020, 11:365. 
7. Falconi M, Giangrossi M, Zabaleta ME, Wang J, Gambini V, Tilio M, Bencardino D, Occhipinti S, Belletti B, Laudadio E, et al: A novel 3'-tRNA(Glu)-derived fragment acts as a tumor suppressor in breast cancer by targeting nucleolin. FASEB J 2019, 33:13228-13240.

8. Luan N, Mu Y, Mu J, Chen Y, Ye X, Zhou Q, Xu M, Deng Q, Hu Y, Tang Z, Wang J: Dicer1 Promotes Colon Cancer Cell Invasion and Migration Through Modulation of tRF-20-MEJB5Y13 Expression Under Hypoxia. Front Genet 2021, 12:638244.

9. Haussecker D, Huang Y, Lau A, Parameswaran P, Fire AZ, Kay MA: Human tRNA-derived small RNAs in the global regulation of RNA silencing. Rna 2010, 16:673-695.

10. Jouinot A, Armignacco R, Assié G: Genomics of benign adrenocortical tumors. J Steroid Biochem Mol Biol 2019, 193:105414.

11. Phan NN, Wang CY, Chen CF, Sun Z, Lai MD, Lin YC: Voltage-gated calcium channels: Novel targets for cancer therapy. Oncol Lett 2017, 14:2059-2074.

12. Tan GC, Negro G, Pinggera A, Tizen Laim NMS, Mohamed Rose I, Ceral J, Ryska A, Chin LK, Kamaruddin NA, Mohd Mokhtar N, et al: Aldosterone-Producing Adenomas: Histopathology-Genotype Correlation and Identification of a Novel CACNA1D Mutation. Hypertension 2017, 70:129-136.

13. Im BH, Rhim H: GABA(B) receptor-mediated ERK1/2 phosphorylation via a direct interaction with $\mathrm{Ca}(\mathrm{V}) 1.3$ channels. Neurosci Lett 2012, 513:89-94.

14. Fourbon Y, Guéguinou M, Félix R, Constantin B, Uguen A, Fromont G, Lajoie L, Magaud C, Lecomte T, Chamorey $\mathrm{E}$, et al: $\mathrm{Ca}(2+)$ protein alpha 1D of $\mathrm{CaV} 1.3$ regulates intracellular calcium concentration and migration of colon cancer cells through a non-canonical activity. Sci Rep 2017, 7:14199.

15. Burotto M, Chiou VL, Lee JM, Kohn EC: The MAPK pathway across different malignancies: a new perspective. Cancer 2014, 120:3446-3456.

16. Guo YJ, Pan WW, Liu SB, Shen ZF, Xu Y, Hu LL: ERK/MAPK signalling pathway and tumorigenesis. Exp Ther Med 2020, 19:1997-2007.

17. Hutton SR, Otis JM, Kim EM, Lamsal Y, Stuber GD, Snider WD: ERK/MAPK Signaling Is Required for Pathway-Specific Striatal Motor Functions. J Neurosci 2017, 37:8102-8115.

18. Santarpia L, Lippman SM, El-Naggar AK: Targeting the MAPK-RAS-RAF signaling pathway in cancer therapy. Expert Opin Ther Targets 2012, 16:103-119.

19. Slattery ML, Mullany LE, Sakoda LC, Wolff RK, Samowitz WS, Herrick JS: The MAPK-Signaling Pathway in Colorectal Cancer: Dysregulated Genes and Their Association With MicroRNAs. Cancer Inform 2018, 17:1176935118766522.

20. Liao X, Li Y: Genetic associations between voltage-gated calcium channels and autism spectrum disorder: a systematic review. Mol Brain 2020, 13:96.

21. Dong X, Fan X, He X, Chen S, Huang W, Gao J, Huang Y, Wang H: Comprehensively Identifying the Key tRNA-Derived Fragments and Investigating Their Function in Gastric Cancer Processes. Onco Targets Ther 2020, 13:10931-10943. 
22. Fu H, Feng J, Liu Q, Sun F, Tie Y, Zhu J, Xing R, Sun Z, Zheng X: Stress induces tRNA cleavage by angiogenin in mammalian cells. FEBS Lett 2009, 583:437-442.

23. Goodarzi H, Liu X, Nguyen HC, Zhang S, Fish L, Tavazoie SF: Endogenous tRNA-Derived Fragments Suppress Breast Cancer Progression via YBX1 Displacement. Cell 2015, 161:790-802.

24. Huang $B$, Yang $H$, Cheng $X$, Wang D, Fu S, Shen $W$, Zhang Q, Zhang L, Xue Z, Li Y, et al: tRF/miR-1280 Suppresses Stem Cell-like Cells and Metastasis in Colorectal Cancer. Cancer Res 2017, 77:31943206.

25. Huang SQ, Sun B, Xiong ZP, Shu Y, Zhou HH, Zhang W, Xiong J, Li Q: The dysregulation of tRNAs and tRNA derivatives in cancer. J Exp Clin Cancer Res 2018, 37:101.

26. Kumar P, Anaya J, Mudunuri SB, Dutta A: Meta-analysis of tRNA derived RNA fragments reveals that they are evolutionarily conserved and associate with AGO proteins to recognize specific RNA targets. BMC Biol 2014, 12:78.

27. Li J, Zhu L, Cheng J, Peng Y: Transfer RNA-derived small RNA: A rising star in oncology. Semin Cancer Biol 2021.

28. Kim HK, Fuchs G, Wang S, Wei W, Zhang Y, Park H, Roy-Chaudhuri B, Li P, Xu J, Chu K, et al: A transfer-RNA-derived small RNA regulates ribosome biogenesis. Nature 2017, 552:57-62.

29. Krishna S, Raghavan S, DasGupta R, Palakodeti D: tRNA-derived fragments (tRFs): establishing their turf in post-transcriptional gene regulation. Cell Mol Life Sci 2021, 78:2607-2619.

30. Tao EW, Wang HL, Cheng WY, Liu QQ, Chen YX, Gao QY: A specific tRNA half, 5'tiRNA-His-GTG, responds to hypoxia via the HIF1alpha/ANG axis and promotes colorectal cancer progression by regulating LATS2. J Exp Clin Cancer Res 2021, 40:67.

31. Kuscu C, Kumar P, Kiran M, Su Z, Malik A, Dutta A: tRNA fragments (tRFs) guide Ago to regulate gene expression post-transcriptionally in a Dicer-independent manner. Rna 2018, 24:1093-1105.

32. Shao Y, Sun Q, Liu X, Wang P, Wu R, Ma Z: tRF-Leu-CAG promotes cell proliferation and cell cycle in non-small cell lung cancer. Chem Biol Drug Des 2017, 90:730-738.

33. Shen Y, Xie Y, Yu X, Zhang S, Wen Q, Ye G, Guo J: Clinical diagnostic values of transfer RNA-derived fragment tRF-19-3L7L73JD and its effects on the growth of gastric cancer cells. J Cancer 2021, 12:3230-3238.

34. Sun C, Fu Z, Wang S, Li J, Li Y, Zhang Y, Yang F, Chu J, Wu H, Huang X, et al: Roles of tRNA-derived fragments in human cancers. Cancer Lett 2018, 414:16-25.

35. Yamasaki S, Ivanov P, Hu GF, Anderson P: Angiogenin cleaves tRNA and promotes stress-induced translational repression. J Cell Biol 2009, 185:35-42.

36. Yang C, Lee M, Song G, Lim W: tRNA(Lys)-Derived Fragment Alleviates Cisplatin-Induced Apoptosis in Prostate Cancer Cells. Pharmaceutics 2021, 13.

37. Zhang M, Li F, Wang J, He W, Li Y, Li H, Wei Z, Cao Y: tRNA-derived fragment tRF-03357 promotes cell proliferation, migration and invasion in high-grade serous ovarian cancer. Onco Targets Ther 2019, 12:6371-6383. 
38. Farina NH, Scalia S, Adams CE, Hong D, Fritz AJ, Messier TL, Balatti V, Veneziano D, Lian JB, Croce $\mathrm{CM}$, et al: Identification of tRNA-derived small RNA (tsRNA) responsive to the tumor suppressor, RUNX1, in breast cancer. J Cell Physiol 2020, 235:5318-5327.

39. Kim HK, Xu J, Chu K, Park H, Jang H, Li P, Valdmanis PN, Zhang QC, Kay MA: A tRNA-Derived Small RNA Regulates Ribosomal Protein S28 Protein Levels after Translation Initiation in Humans and Mice. Cell Rep 2019, 29:3816-3824 e3814.

40. Wang T, Cao L, He S, Long K, Wang X, Yu H, Ma B, Xu X, Li W: Small RNA sequencing reveals a novel tsRNA-06018 playing an important role during adipogenic differentiation of hMSCs. J Cell Mol Med 2020, 24:12736-12749.

41. Jin F, Yang L, Wang W, Yuan N, Zhan S, Yang P, Chen X, Ma T, Wang Y: A novel class of tsRNA signatures as biomarkers for diagnosis and prognosis of pancreatic cancer. Mol Cancer 2021, 20:95.

42. Zhu L, Li J, Gong Y, Wu Q, Tan S, Sun D, Xu X, Zuo Y, Zhao Y, Wei YQ, et al: Exosomal tRNA-derived small RNA as a promising biomarker for cancer diagnosis. Mol Cancer 2019, 18:74.

43. Wang X, Zhang Y, Ghareeb WM, Lin S, Lu X, Huang Y, Huang S, Xu Z, Chi P: A Comprehensive Repertoire of Transfer RNA-Derived Fragments and Their Regulatory Networks in Colorectal Cancer. $J$ Comput Biol 2020, 27:1644-1655.

44. Balatti V, Nigita G, Veneziano D, Drusco A, Stein GS, Messier TL, Farina NH, Lian JB, Tomasello L, Liu CG, et al: tsRNA signatures in cancer. Proc Natl Acad Sci U S A 2017, 114:8071-8076.

\section{Tables}

Table 1 Pathological association between the tRF-Val-CAC-016 expression and the clinicopathological features in $\mathbf{4 0}$ pairs of GC tissues. 


\begin{tabular}{|c|c|c|c|c|c|}
\hline \multicolumn{2}{|c|}{ Characteristics } & \multirow[t]{2}{*}{ Case } & \multicolumn{2}{|c|}{ tRF-Val-CAC-016 expression } & \multirow[t]{2}{*}{ p-value } \\
\hline & & & Low & High & \\
\hline All & & 40 & 25 & 15 & \\
\hline \multirow[t]{2}{*}{ Age (years) } & $<63$ & 15 & 9 & 6 & 0.800 \\
\hline & $>=63$ & 25 & 16 & 9 & \\
\hline \multirow[t]{2}{*}{ Gender } & Male & 33 & 22 & 11 & 0.237 \\
\hline & Female & 7 & 3 & 4 & \\
\hline \multirow[t]{2}{*}{ Size $(\mathrm{cm})$} & $<6.0$ & 21 & 9 & 12 & $0.007^{\star \star}$ \\
\hline & $>=6.0$ & 19 & 16 & 3 & \\
\hline \multirow[t]{2}{*}{ Histology } & well-differentiated & 16 & 6 & 10 & $0.008^{\star *}$ \\
\hline & poor-differentiated & 24 & 19 & 5 & \\
\hline \multirow[t]{2}{*}{ TNM stage } & $\mathrm{H}$ & 10 & 6 & 4 & 0.850 \\
\hline & III-IV & 30 & 19 & 11 & \\
\hline
\end{tabular}

${ }^{*} p<0.05,{ }^{* *} p<0.01$

\section{Figures}

\section{Figure 1}

tRFs and tiRNAs sequencing profiles of GC tissues. (a) Heatmap of the differentially expressed tsRNAs (DETs). (b) A total of 69 up-regulated and 42 down-regulated DETs and 277 NDETs were presented using the volcano plot, and five down-regulated and six up-regulated tsRNAs were obviously shown in the plot based on the foldchange (FC) of the profiles $\left(\log _{2} F C>=3\right.$ or $\left.\log _{2} F C<=-3, p<0.05\right)$. (c) Heatmap of the correlation coefficient. The color in the panel represents the correlation coefficient of the two samples. Blue represents a high correlation coefficient between the two samples, and white indicates the low similarity of the two samples.

\section{Figure 2}


tRF-Val-CAC-016 was significantly low-expressed in GC tissues. (a-b) Gel-electrophoresis was applied to verify the PCR product of tRF-Val-CAC-016, and the Sanger sequencing was undertaken to confirm the results. (c) Fluorescent In Situ Hybridization (FISH) assay indicated that tRF-Val-CAC-016 is located in both nuclei and cytoplasm, and mainly in the cytoplasm. (d) The expression levels of tRF-Val-CAC-016 were tested in GC cell lines, NCI-N87 and HGC-27 were finally selected. (e) tRF-Val-CAC-016 mimics could significantly increase the expression level of tRF-Val-CAC-016. (f) The low expression level of tRF-ValCAC-016 was confirmed in 40 pairs of GC tissues. (g) Correlation between the expression level of tRF-ValCAC-016 and the long-term outcomes (overall survival, OS) of GC was not significant $(P=0.5802)$. ${ }^{P}<$ $0.05,{ }^{\star} \mathrm{P}<0.01$, statistically significant. Scale bar $=10 \mu \mathrm{m}$.

\section{Figure 3}

tRF-Val-CAC-016 suppressed the proliferation of GC. (a-b) CCK-8 assays for tRF-Val-CAC-016 mimics, tRFVal-CAC-016 inhibitor, and oxaliplatin. (c-d) The ethynyl-2'-deoxyuridine (EdU) assays demonstrated that tRF-Val-CAC-016 could suppress the cell replication activity, but tRF-Val-CAC-016 inhibitor enhanced the replication activity of GC. (e-g) tRF-Val-CAC-016 adjusted the S phase significantly both in NCI-N87 and HGC-27. (h-i) tRF-Val-CAC-016 modulated the viability of GC cells in colony formation assays. (j-k) Immunoblotting assays for CyclinD1, CyclinB, c-myc. ${ }^{*} \mathrm{P}<0.05$, ${ }^{* *} \mathrm{P}<0.01$, statistically significant.

\section{Figure 4}

GO and KEGG enrichment analysis of sequencing profiles. (a-d) GO and KEGG enrichment analysis for the target genes of six down-regulated tsRNAs (tRF-Val-CAC-016, tRF-Glu-TTC-027, tRF-Glu-TTC-026, tRFSer-TGA-011, tiRNA-Pro-TGG-001, tRF-Ser-GCT-113). (e-h) GO and KEGG enrichment analysis for the four up-regulated tsRNAs (tiRNA-Val-CAC-001, tiRNA-His-GTG-001, tRF-Glu-TTC-017, tiRNA-Asp-GTC-001).

\section{Figure 5}

CACNA1d was verified up-regulated in GC tissues and was selected as the potential target of tRF-Val-CAC016. (a) The Venn diagram took the overlap of MAPK components and the predicted target genes of tRFVal-CAC-016. (b-c) CACNA1d, PLA2G4A, and TNF in GSE65801, CACNA1d, and PLA2G4A in TCGA-STAD were significantly up-regulated. (d-l) CACNA1d, TNF, TGFBR1, PDGFC, GADD45B were significantly and negatively related to the prognosis of GC. (m-n) The tissue microarray (TMA) with 90 pairs of GC specimens, including detailed follow-up data. (o) The expression of CACNA1d was not significantly related to the prognosis of GC ( $p=0.1805)$. (p) Ten representative IHC images of TMA were presented, including GC and NATS. (q) The protein levels of CACNA1d were highly expressed in nine GC tissues 
compared with corresponding NATs. (r) si-CACNA1d-1 was a better inhibitor for CACNA1d compared with si-CACNA1d-2 and si-CACNA1d-3. (s) pcDNA-CACNA1d could significantly enhance the expression of CACNA1d. (t) The tRF-Val-CAC-016 inhibitor was able to reverse the suppressive function of si-CACNA1d on GC cells to some extent. (u) The effect of pcDNA-CACNA1d on GC was partly relieved by tRF-Val-CAC016 mimics. ${ }^{*} \mathrm{P}<0.05, * * \mathrm{P}<0.01$, statistically significant.

\section{Figure 6}

tRF-Val-CAC-016 was immunoprecipitated by Argonaute-2 and could modulate the proliferation of GC by targeting CACNA1d. (a) Binding relation between tRF-Val-CAC-016 and CACNA1d mRNA. (b-c) tRF-ValCAC-016 mimics could significantly reduce the expression of CACNA1d using RT-PCR and immunoblotting. (d) Dual-luciferase reporter assay indicated that WT-CACNA1d-3'UTR plus tRF-Val-CAC016 mimics group could significantly reduce the luciferase ratio compared with other groups in NCl-N87 and HGC-27. (e) In RIP assay, tRF-Val-CAC-016 was significantly immunoprecipitated by Argonaute-2 compared with the IgG group. (f-g) Gel-electrophoresis further verified the PCR product of RIP assays, and the immunoblotting confirmed the integrity of the process to wash the magnetic beads. ${ }^{*} \mathrm{P}<0.05$, ${ }^{*} \mathrm{P}<$ 0.01 , statistically significant.

\section{Figure 7}

si-CACNA1d inhibited the proliferation of GC and could be rescued by tRF-Val-CAC-016 inhibitor. (a-b) In the CCK-8 assays, si-CACNA1d could significantly decline the proliferation of GC cells and was partly recovered by tRF-Val-CAC-016 inhibitor in HGC-27 and NCI-N87. (c-d) In the EdU assays, the tRF-Val-CAC016 inhibitor could rescue the inhibitory effect of si-CACNA1d in terms of cell replication activity. (e-g) siCACNA1d resulted in the $\mathrm{G} 1$ phase arrest in $\mathrm{HGC}-27$, but $\mathrm{S}$ phase arrest in NCl-N87 with flow cytometry and could both be rescued by tRF-Val-CAC-016 inhibitor. (h-i) The rescue effect of the tRF-Val-CAC-016 inhibitor on si-CACNA1d was also confirmed in the colony formation assays. ( $\mathrm{j}-\mathrm{k}$ ) The suppressive function of si-CACNA1d on the protein expression of CACNA1d, CyclinD1, CyclinB, c-myc was partly resumed by tRF-Val-CAC-016 inhibitor. ${ }^{*} \mathrm{P}<0.05,{ }^{\star *} \mathrm{P}<0.01$, statistically significant.

\section{Figure 8}

pCDNA-CACNA1d strengthened the proliferation of GC and was modulated by tRF-Val-CAC-016 mimics. tRF-Val-CAC-016 mimics could significantly reverse the reinforced effect of pcDNA-CACNA1d on the proliferation of GC cells in terms of the CCK-8 (a-b), EdU (c-d), flow cytometry for cell cycle (e-g) and colony formation assays (h-i). (j-k) pcDNA-CACNA1d significantly lifted the protein levels of CACNA1d, 
CyclinD1, CyclinB, c-myc, and tRF-Val-CAC-016 mimics could decline this function. ${ }^{*} \mathrm{P}<0.05,{ }^{* \star P}<0.01$, statistically significant.

\section{Figure 9}

Inhibitor of MAPK signaling pathway (p38 MAPK-IN) significantly reversed the enhancement of tRF-ValCAC-016 inhibitor on GC proliferation. (a-b) tRF-Val-CAC-016 inhibitor enhanced the GC proliferation in the CCK-8 assay, which p38 MAPK-IN could partly reverse. (c-d) tRF-Val-CAC-016 inhibitor promoted the cell replication activity, and p38 MAPK-IN could decline this activity in HGC-27 and NCI-N87. The reversal phenomena also happened in the flow cytometry assays for cell cycle (e-g) and immunoblotting assays for CACNA1d, CyclinD1, CyclinB, c-myc (h-i). ${ }^{*} \mathrm{P}<0.05,{ }^{* *} \mathrm{P}<0.01$, statistically significant.

\section{Figure 10}

tRF-Val-CAC-016 inhibited tumor proliferation in the NCl-N87 xenografts. (a) Representative images of $\mathrm{NCl}-\mathrm{N} 87$ xenografts in vivo. Six mice are included in each group. (b-d) The results indicated that tRF-ValCAC-016 declined tumor growth capacity in mice in the aspect of the bodyweight of the xenografts and the tumor volumes. (e) The PCR results of these resected tumors showed that tRF-Val-CAC-016 agomir significantly increased the expression of tRF-Val-CAC-016 compared with the agomir control group and normal saline (NS) group. (f) The immunoblotting assays showed the inhibitory effect of tRF-Val-CAC016 agomir on CACNA1d, c-myc, and cyclinD1. (g-h) The immunofluorescence staining assays for ki-67 and CACNA1d. (i) The Immunohistochemistry (IHC) assays demonstrated the low expression of CACNA1d in the tRF-Val-CAC-016 agomir group compared with the agomir control group and normal saline (NS) group. ${ }^{*} \mathrm{P}<0.05,{ }^{*} \mathrm{P}<0.01$, statistically significant.

\section{Figure 11}

\section{tRNA derivative tRF-Val-CAC-016 modulates the canonical MAPK signaling pathway by targeting}

CACNA1d. (a) The mechanism diagram clearly explained the pattern that tRF-Val-CAC-016 exerted its influence on the MAPK signaling pathway. (b) In the immunoblotting assays of NCI-N87, tRF-Val-CAC-016 mimics suppressed the expression of CACNA1d (Cav1.3), ERK, p-ERK, and p-p38. tRF-Val-CAC-016 inhibitor enhanced the expression of CACNA1d (Cav1.3), ERK, p-JNK, p38, cyclinB, cyclinD1, c-myc. (c) siCACNA1d could inhibit the expression levels of CACNA1d (Cav1.3), JNK, p-p38, cyclinD1, and c-myc, which was able to be reversed by tRF-Val-CAC-016 inhibitor. (d) pcDNA-CACNA1d increased the expression of CACNA1d (Cav1.3), ERK, p-ERK, p-JNK, p38, p-p38, cyclinB, cyclinD1, and c-myc, which was rescued by tRF-Val-CAC-016 mimics. (e) In HGC-27, tRF-Val-CAC-016 mimics declined, but tRF-Val-CAC- 
016 inhibitor promoted the protein levels of CACNA1d (Cav1.3), ERK, p-ERK, JNK, p-JNK, p38, p-p38, cyclinB, cyclinD1, c-myc. (f) CACNA1d (Cav1.3), ERK, JNK, p-JNK, p38, p-p38, cyclinB, and cyclinD1 were down-regulated by si-CACNA1d, and this effect was reversed by tRF-Val-CAC-016 inhibitor. (g) CACNA1d (Cav1.3), ERK, p-ERK, JNK, p-JNK, p38, p-p38, cyclinB, cyclinD1, and c-myc were enhanced by pcDNACACNA1d, which could be deteriorated by tRF-Val-CAC-016 mimics.

\section{Supplementary Files}

This is a list of supplementary files associated with this preprint. Click to download.

- SupplementaryFigure1.tif

- SupplementaryFigure2.tif

- SupplementaryFigure3.tif

- SupplementaryFigure4.tif

- Supplementarytable1.docx

- Supplementarytable2.docx 